\title{
Design and Implementation of the adaptive CCCII-OTA Filter
}

$$
\text { Sheng-qian } \mathrm{Ma}^{1, \mathrm{a}^{*}} \text {, Yan-ping } \mathrm{Ji}^{2, \mathrm{~b}} \text {, Zhi-feng } \mathrm{Ma}^{3, \mathrm{c}} \text { Li-rong Zheng }{ }^{4, \mathrm{~d}}
$$

${ }^{1}$ College of Physics and Electronic Engineering, Northwest Normal University, Lanzhou, China

${ }^{2}$ College of Physics and Electronic and Mechanical Engineering, Hexi University, Zhangye, China

${ }^{3}$ College of Zhixing, Northwest Normal University, Lanzhou, China

${ }^{4}$ College of Physics and Electronic Engineering, Northwest Normal University, Lanzhou, China

aemail: s.q.ma@163.com, bemail: 416894253@qq.com,

cemail: mzf_10@126.com, 'email: 1203822534@qq.com

Keywords: Adaptive filter; multifunction filtering; frequency-current converter circuit (F/I); current-controlled filter; CCCII-OTA.

Abstract. This paper proposes an adaptive filter structure and implementation method which based on operational transconductance amplifier (OTA) and the current controlled conveyor (CCCII). After preprocessing and shaping, it can generate a frequency signal, which be converted into current signal $I_{\mathrm{B}}$ by the $\mathrm{F} / \mathrm{I}$ circuit. $I_{\mathrm{B}}$ is as the bias current of the filter to control the filtering frequency. The multifunction filter current-controlled is constructed using two CCCII, one OTA, and some capacitors. The filter has low-pass, high-pass, and band-pass function. So that frequency of the filter can automatically track the input signal frequency. This paper describes the principles, the composition, design and implementation of adaptive CCCII-OTA filter. When the frequency of the input signal changes at a wide range from $10 \mathrm{kHz}$ to $5 \mathrm{MHz}$, the theoretical values of the filter are in good agreement with the experimental results.

\section{Introduction}

Adaptive filter has a wide range of applications [1]. So far, the study of adaptive filter is centered on two aspects: digital filter and analog filter. Digital adaptive filters are accomplished mainly through DSP or FPGA and other hardware platform [2, 3]. However, its processing speed can't satisfy the high frequency application. Analog adaptive filters have two implementation methods. One method is to use existing monolithic filter chip, such as MAX26X, MAX29X, which combines switched capacitor filter with phase-locked loop technology [4, 5], or controls frequency of filter by changing the clock frequency. Another method is to use frequency-voltage converter, which combines analog multiplier and current feedback operational amplifier. It uses voltage to control the filter so as to track filter's frequency $[6,7]$.

Aiming at the filter signal processing speed is not high, and the scope of the frequency tracking is narrower and other shortcomings, some improvement methods were proposed [8,9]. The CCCII and OTA are used widely in the design of filter $[10,11]$. This paper proposes an adaptive filter structure which based on the F/I circuit and CCCII-OTA. The input signal is amplified and shaped. It is converted into a current signal by F/I circuit. The key point is based on the CCCII-OTA to design a filter whose filtering frequency can be controlled by the current. It discusses the principles, design, and implement method of the filter.

\section{The system composition and working principle}

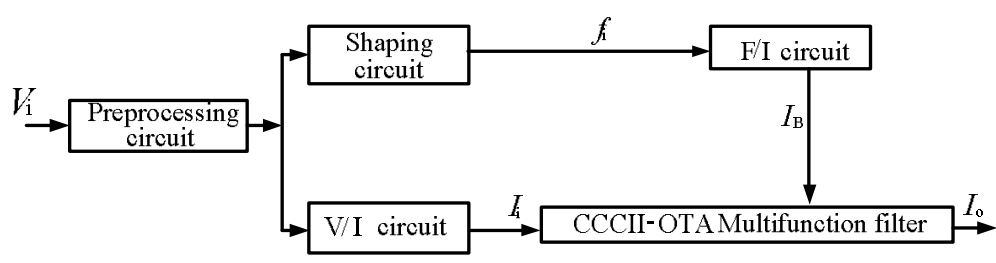

Fig. 1 The system structure diagram 
The system structure diagram of the adaptive filter is shown in Fig.1. $V_{\mathrm{i}}$ is the input signal, Io is the output of the filter. The amplifying circuit amplifies the input voltage signal for shaping and V/I circuit using. The shaping circuit can convert the input signal into a corresponding square wave with the same frequency $f_{\mathrm{i}} ; \mathrm{F} / \mathrm{I}$ circuit converts $f_{\mathrm{i}}$ to a output current $I_{\mathrm{B}}$. It has a linear relationship between $f_{\mathrm{i}}$ and $I_{\mathrm{B}}$, and it can indirectly obtain the frequency of the input signal by measuring $I_{\mathrm{B}}$. Thus it achieved a DC current which varies with the frequency of the input $\mathrm{AC}$ signal. The $I_{\mathrm{B}}$ is as the bias current of multifunction filter which is composed by CCCII, OTA, and some capacitors. The filter's frequency is controlled by its bias current $I_{\mathrm{B}}$.

In Fig. 1 by other route signal through V/I circuit which is converted the voltage signal into a corresponding current signal. Then it inputs directly into the filter designed.

So in Fig. 1 the input signal is amplified and shaped. It is converted into a current signal $I_{\mathrm{B}}$ by F/I circuit. $I_{\mathrm{B}}$ is as the bias current of the filter to control the filtering frequency. That is, the filtering frequency can follow the input signal frequency change. So as it achieves adaptive filtering function.

\section{Frequency-current converter circuit}

Signal conditioning circuit mainly includes amplifying circuit and limiting circuit. It consists of AD811, capacitors and resistors. It is used to amplify a weak input signal for shaping circuit using. Its frequency range is up to $30 \mathrm{MHz}$.

Since the F/I circuit requires a pulse signal, so it needs to shape the input signal. The shaping circuit is shown in Fig. 2, which is composed of MAX903, capacitors and resistors. $V_{\text {ref }}$ is the reference voltage, $V_{\mathrm{i}}$ is the input signal, $V_{1}$ is the output signal and it is as the input signal of $\mathrm{F} / \mathrm{V}$.

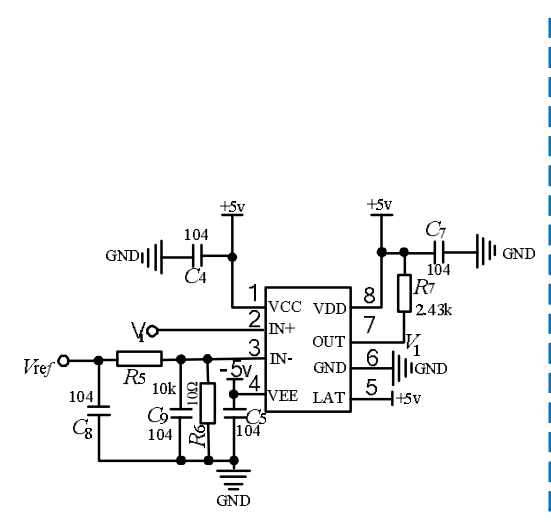

Fig. 2 Shaping circuit

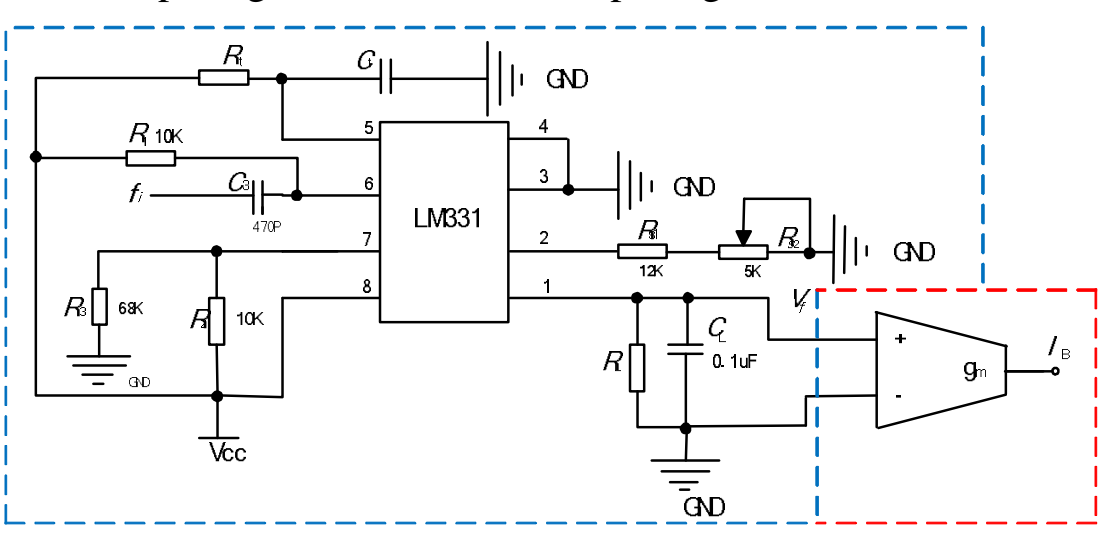

Fig. 3 Frequency current conversion circuit

The F/I circuit is shown in Fig. 3. Frequency-current circuit can convert the input pulse signal into a current signal. It is consisted by a dedicated F/V chip LM331, OTA and a small amount of capacitors and resistors. In which F/V circuit is shown in blue dotted line, and the red portion is voltage to current conversion circuit. The voltage output signal of LM331 $V_{\mathrm{f}}$ is added to OTA, and the final output is a current signal. The output voltage $V_{\mathrm{f}}$ and the input frequency $f_{\mathrm{i}}$ can be expressed as:

$$
V_{f}=\left(2.09 R_{\mathrm{L}} R_{\mathrm{t}} C_{\mathrm{t}} f_{\mathrm{i}}\right) / R_{\mathrm{s}} .
$$

Where $R_{\mathrm{s}}=R_{\mathrm{s} 1}+R_{\mathrm{s} 2}$. According to the OTA relationship between voltage and current, $I_{\mathrm{B}}$ is the product of $V_{\mathrm{f}}$ and transconductance gain $g_{\mathrm{m}}$, to combine Eq.1 it can obtain:

$$
I_{\mathrm{B}}=\left(2.09 R_{\mathrm{L}} R_{\mathrm{t}} C_{\mathrm{t}} g_{m} f_{\mathrm{i}}\right) / R_{\mathrm{s}} \text {. }
$$

As long as a reasonable adjustment capacitance, resistance values, Eq. 2 shows that the output current $I_{\mathrm{B}}$ and input frequency $f_{\mathrm{i}}$ has a linear relationship.

\section{CCCII-OTA-based second-order filter design}

Current-mode circuit of CCCII-OTA can solve some problems of the voltage-mode circuit. It has better performance in the dynamic range, bandwidth, speed, and so on. 
Second-order filter not only can be applied directly, but also can be cascaded as high-order filters. Second-order circuits are often called double integral circuit, which can obtain all-pass filter and band-stop filter by using high-pass output, low pass output and band-pass output. Also, it can achieve multi-functional filtering through combining high-pass, low-pass and band-pass filter's transfer functions. The filter composed by CCCII-OTA which is shown in Fig. 4.

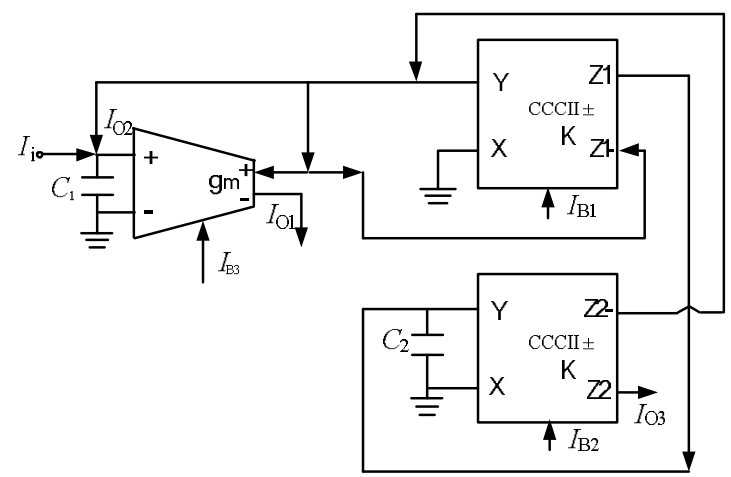

Fig. 4 Filter consisting of CCCII-OTA

To use port characteristics CCCII-OTA, it can get the following equations:

$$
\begin{aligned}
& H_{\mathrm{BP}}(S)=\frac{I_{\mathrm{O} 1}}{I_{\mathrm{i}}}=\frac{S C_{2} R_{\mathrm{X} 2} R_{\mathrm{X} 1}}{S^{2} C_{1} C_{2} R_{\mathrm{X} 1} R_{\mathrm{X} 2}+S C_{2} R_{\mathrm{X} 2}\left(1+g_{\mathrm{m}} R_{\mathrm{X} 1}\right)+1} . \\
& H_{\mathrm{HP}}(S)=\frac{I_{\mathrm{O} 2}}{I_{\mathrm{i}}}=\frac{S^{2} C_{1} C_{2} R_{\mathrm{X} 2} R_{\mathrm{X} 1}}{S^{2} C_{1} C_{2} R_{\mathrm{X} 1} R_{\mathrm{X} 2}+S C_{2} R_{\mathrm{X} 2}\left(1+g_{\mathrm{m}} R_{\mathrm{X} 1}\right)+1} . \\
& H_{\mathrm{LP}}(S)=\frac{I_{\mathrm{O} 3}}{I_{\mathrm{i}}}=\frac{1}{S^{2} C_{1} C_{2} R_{\mathrm{X} 1} R_{\mathrm{X} 2}+S C_{2} R_{\mathrm{X} 2}\left(1+g_{\mathrm{m}} R_{\mathrm{X} 1}\right)+1} .
\end{aligned}
$$

By the Eq.3 5, it can obtain:

$$
\begin{aligned}
& \omega_{\mathrm{o}}=\sqrt{1 /\left(C_{1} C_{2} R_{\mathrm{X} 2} R_{\mathrm{X} 1}\right)} . \\
& Q=1 /\left(1+g_{\mathrm{m}} R_{\mathrm{X} 1}\right) \sqrt{\left(C_{1} R_{\mathrm{X} 1}\right) /\left(C_{2} R_{\mathrm{X} 2}\right)} .
\end{aligned}
$$

The $\omega_{\mathrm{o}}$ is filtering frequency, $Q$ is quality factor. If $R_{\mathrm{X} 1}=R_{\mathrm{X} 2}=R_{\mathrm{X}}, C_{1}=C_{2}=C$, The Eq.6 and Eq.7 can be written as:

$$
\begin{aligned}
& \omega_{\mathrm{o}}=1 /\left(R_{\mathrm{X}} C\right) . \\
& Q=1 /\left(1+g_{m} R_{\mathrm{X}}\right) .
\end{aligned}
$$

To adjust the value of $Q$ by $C_{1}$ and $C_{2}, \omega_{\mathrm{o}}$ can be controlled by $R_{\mathrm{X}}$, and $R_{\mathrm{X}}$ can be controlled by the bias current, therefore $\omega_{\mathrm{o}}$ and $Q$ can be adjusted independently. $V_{\mathrm{T}}=26 \mathrm{mV}, R_{\mathrm{X}}$ is parasitic resistance of $\mathrm{X}$ port of CCCII. By the formula:

$$
\begin{aligned}
& R_{\mathrm{X}}=V_{\mathrm{T}} / 2 I_{\mathrm{B}} . \\
& f_{\mathrm{o}}=\omega /(2 \pi)=I_{\mathrm{B}} /\left(\pi V_{\mathrm{T}} C\right) .
\end{aligned}
$$

By the Eq. 2 and Eq.11, it can be seen that changing $I_{\mathrm{B}}$ can effectively change the output frequency $f_{\mathrm{o}}$. It is able to achieve frequency adaptive function.

$$
f_{\mathrm{o}}=\omega /(2 \pi)=I_{B} /\left(\pi V_{T} C\right)=\left(2.09 R_{\mathrm{L}} R_{\mathrm{t}} C_{\mathrm{t}} g_{m} f_{i}\right) /\left(\pi V_{T} C R_{\mathrm{s}}\right) .
$$

If suitable parameters of resistors and capacitors be selected, then it can get: 


$$
f_{\mathrm{o}}=f_{\mathrm{LP}}=f_{\mathrm{HP}}=f_{\mathrm{BP}}=f_{\mathrm{i}} \text {. }
$$

The Eq.13 shows that the frequency of the filter designed can follow input signal change. That is the filter has adaptive tracking ability.

\section{Experimental results}

The circuit structure of second-order adaptive filter was made of double-sided PCB. It carried out the actual board fabrication and measurement. The power voltage is $\pm 5 \mathrm{~V}$, PMOS and NMOS transistors used are IRF9530NPBF and SVF2N60F. The parameters are selected, $R_{\mathrm{t}}=910 \Omega, R_{\mathrm{L}}=$ $762 \Omega, R_{\mathrm{s}}=14.75 \mathrm{k} \Omega, C_{\mathrm{t}}=10 \mathrm{nF}, g_{\mathrm{m}}=249.6 \mathrm{uS}, C=3 \mathrm{nF}$. The current amplitude and frequency of the test point can be recorded by observing the output waveform of band-pass filter through adjusting the frequency of signal source. The comparison of measurement and theoretical amplitude-frequency characteristic curves is shown in Fig. 5. The theoretical values were simulated by Cadence16.3.
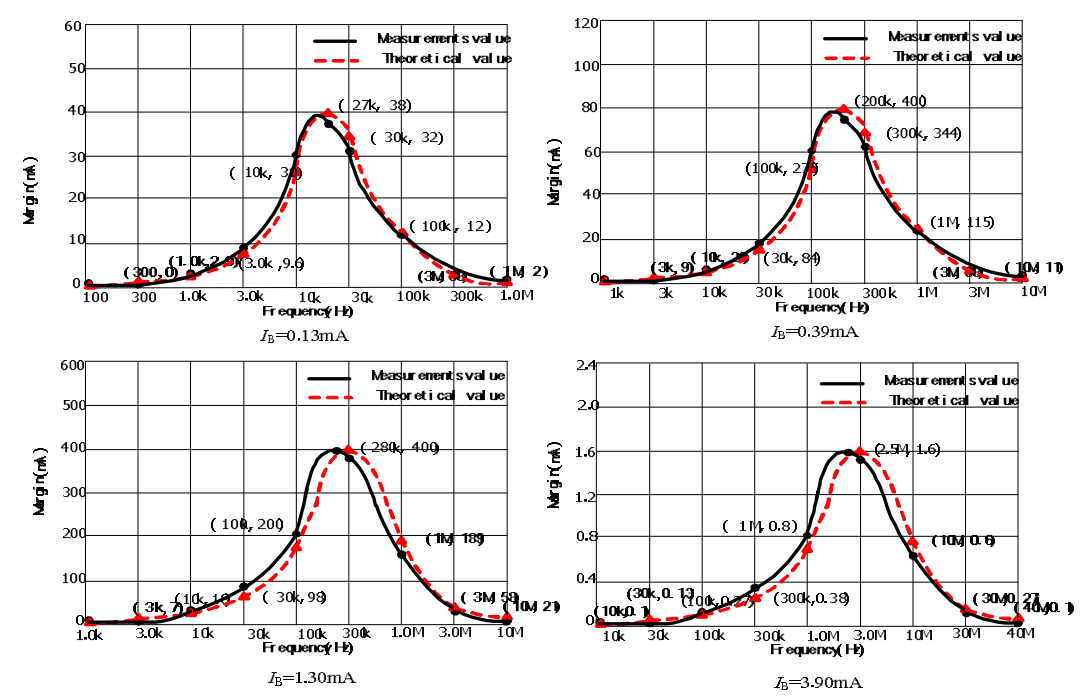

Fig. 5 The frequency response of band-pass filter

Table 1 shows second-order CCCII-OTA adaptive band-pass filter test results. In which $f_{\mathrm{i}}$ is the signal frequency and $f_{\mathrm{o}}$ is the center frequency of the filter designed. The frequency relative error $\delta_{\mathrm{f}}$ is not more than $4.0 \%$. It shows that the filter designed has frequency tracking function. That is the frequency of the filter can follow the input signal to change, the filter has an adaptive ability. For the space limiting only band-pass filter experimental results are given. In fact, for low-pass, high-pass filtering function test, the conclusion is as same.

Table 1 The test results of adaptive band-pass filter

\begin{tabular}{lllll}
\hline$I_{\mathrm{B}}[\mathrm{mA}]$ & 0.13 & 0.39 & 1.30 & 3.90 \\
$f_{\mathrm{i}}[\mathrm{Hz}]$ & $27 \mathrm{k}$ & $200 \mathrm{k}$ & $280 \mathrm{k}$ & $2.50 \mathrm{M}$ \\
$f_{\mathrm{o}}[\mathrm{Hz}]$ & $26 \mathrm{k}$ & $197 \mathrm{k}$ & $274 \mathrm{k}$ & $2.47 \mathrm{M}$ \\
\hline$\delta_{\mathrm{f}}[\%]$ & 3.7 & 1.5 & 2.1 & 1.2 \\
\hline
\end{tabular}

\section{Conclusions}

The method of the filter designed and implementation is described. By the amplifying, shaping, and $\mathrm{F} / \mathrm{I}$ circuit the input signal is converted into a DC current $I_{\mathrm{B}}$ which has a linear relationship with signal frequency $f_{\mathrm{i}}$. The current is used as bias current to control the frequency of multifunction filter which is constructed based on the CCCII-OTA. The transfer function of the filter is derived. Because frequency of the filter can be controlled by changing bias current of CCCII-OTA, so it is able to get filter's frequency tracking and to achieve adaptive filtering. The scheme is proposed, at the same time, 
the hardware circuit is designed. The test results for band-pass function of the filter designed are in good agreement with the theoretical value, it shows that the filter can automatically track signal frequency. And it proved the correctness and feasibility scheme of the filter design.

\section{Acknowledgment}

Authors would like to acknowledge the support of the National Science Foundation of China (NSFC) under grant NO. 61162017, and and the Postgraduate Tutor Project of Universities of Gansu Province: NO.1101-03.

\section{References}

[1] Ignacio E. Parra, Wilmar Hernandez, Eduardo Fernandez. On the convergence of LMS filters under periodic signals, J. Digital Signal Processing. 23 (2013) 808-816.

[2] Jiangnan Yuan, Tangbi Yu, Huihuang Chen. Based AccelDSP adaptive filter design, J. Journal of Xiamen University. 49 (2010) 205-209.

[3] Yiyong Lin, Zheng Zheng, Zhihui Zhao. Ultrasound RF signals such as an adaptive filter based on FPGA, J. Beijing Biomedical Engineering. 31 (2012) 21-25.

[4] Weiwei Yu, Detian Yan, Yue Yang engineering design and application of automatic tracking filter MAX260, J. Based on Modern Electronic Technology. 5 (2008) 76-77.

[5] Limin Tao, Yue Li, Xisen Wen. Filtering method based switched capacitor signal tracking technology and its application in Rotor, J. China Mechanical Engineering. 18 (2007) 427-430.

[6] Lixia Wang, Xiaohui Cheng, Gongyou Min. LM331 based on the frequency meter, J. Instrument Technique and Sensor. 9(2007) 23-26.

[7] Shengqian Ma, Yanping Ji, Xingping Ran, Weizhao Zhang. Design of the voltage-controlled bandpass filter based on current feedback Op., J. Advanced Materials Research. 986-987 (2014) 1081-1085.

[8] Weizhao Zhang, Shengqian Ma and Xingping Ran. Design of the broadband programmed second-order lower-pass filter. Application of Electronic Technique. 7 (2011)97-100.

[9] Chan S C, Zou Y X. A recursive least M-estimate algorithm or robust adaptive filtering in impulsive noise: fast algorithm and convergence performance analysis, J. Signal Processing, IEEE Transactions on. 52 (2004) 975-991.

[10] Yuce E, Minaei S, Cicekoglu O. Universal current-mode active-C filter employing minimum number of passive elements, J. Analog Integrated Circuits and Signal Processing. 46(2006) 169-171.

[11] Applebuam S P, Chapman D J. Adaptive arrays with main beam constrains, J. IEEE, Trans. Special Issue on Adaptive Antennas. AP24 (1976) 650-662. 